University of Wollongong

Research Online

Australian Institute for Innovative Materials -

Papers

Australian Institute for Innovative Materials

$1-1-2017$

BN Nanosheet/Polymer Films with Highly Anisotropic Thermal Conductivity for Thermal Management Applications

\author{
Yuanpeng Wu \\ Deakin University \\ Ye Xue \\ Rowan University \\ Si Qin \\ Deakin University \\ Dan Liu \\ Deakin University \\ Xuebin Wang \\ Nanjing University
}

See next page for additional authors

Follow this and additional works at: https://ro.uow.edu.au/aiimpapers

Part of the Engineering Commons, and the Physical Sciences and Mathematics Commons

Research Online is the open access institutional repository for the University of Wollongong. For further information contact the UOW Library: research-pubs@uow.edu.au 


\title{
BN Nanosheet/Polymer Films with Highly Anisotropic Thermal Conductivity for Thermal Management Applications
}

\author{
Abstract \\ The development of advanced thermal transport materials is a global challenge. Two-dimensional \\ nanomaterials have been demonstrated as promising candidates for thermal management applications. \\ Here, we report a boron nitride (BN) nanosheet/polymer composite film with excellent flexibility and \\ toughness prepared by vacuum-assisted filtration. The mechanical performance of the composite film is \\ highly flexible and robust. It is noteworthy that the film exhibits highly anisotropic properties, with superior \\ in-plane thermal conductivity of around $200 \mathrm{~W} \mathrm{~m} \mathrm{-1} \mathrm{K} \mathrm{-1} \mathrm{and} \mathrm{extremely} \mathrm{low} \mathrm{through-plane} \mathrm{thermal}$ \\ conductivity of $1.0 \mathrm{~W} \mathrm{m-1} \mathrm{K-1,} \mathrm{making} \mathrm{this} \mathrm{material} \mathrm{an} \mathrm{excellent} \mathrm{candidate} \mathrm{for} \mathrm{thermal} \mathrm{management} \mathrm{in}$ \\ electronics. Importantly, the composite film shows fire-resistant properties. The newly developed \\ unconventional flexible, tough, and refractory BN films are also promising for heat dissipation in a variety \\ of applications. \\ Disciplines \\ Engineering | Physical Sciences and Mathematics

\section{Publication Details} \\ Wu, Y., Xue, Y., Qin, S., Liu, D., Wang, X., Hu, X., Li, J., Wang, X., Bando, Y., Golberg, D., Chen, Y., Gogotsi, Y. \& \\ Lei, W. (2017). BN Nanosheet/Polymer Films with Highly Anisotropic Thermal Conductivity for Thermal \\ Management Applications. ACS Applied Materials and Interfaces, 9 (49), 43163-43170.

\section{Authors} \\ Yuanpeng Wu, Ye Xue, Si Qin, Dan Liu, Xuebin Wang, Xiao Hu, Jingliang Li, Xungai Wang, Yoshio Bando, \\ Dmitri Golberg, Ying I. Chen, Yury Gogotsi, and Weiwei Lei
}




\section{BN nanosheet/polymer films with highly}

\section{anisotropic thermal conductivity for thermal}

\section{management application}

Yuanpeng $\mathrm{Wu}^{1,2}$, Ye Xue ${ }^{3}$, Si Qin ${ }^{1}$, Dan $\mathrm{Liu}^{1}$, Xuebin Wang ${ }^{4,6}, \mathrm{Xiao} \mathrm{Hu}^{3}$, Jingliang $\mathrm{Li}^{1}$, Xungai Wang $^{1}$, Yoshio Bando ${ }^{4}$, Dmitri Golberg ${ }^{4,7}$, Ying Chen $^{1}$, Yury Gogotsi ${ }^{5}$, Weiwei Lei ${ }^{1 *}$

${ }^{1}$ Institute for Frontier Materials, Deakin University, Waurn Ponds Campus, Locked Bag 20000, Victoria 3220, Australia

2 School of Materials Science and Engineering, Southwest Petroleum University, Chengdu 610500, China

${ }^{3}$ Department of Physics and Astronomy and Department of Biomedical Engineering, Rowan University, 201 Mullica Hill Road, Glassboro, New Jersey 08028, United States

${ }^{4}$ International Center for Materials Nanoarchitectonics (WPI-MANA), National Institute for Materials Science (NIMS), Namiki 1-1, Tsukuba, Ibaraki 305-0044, Japan

5 A. J. Drexel Nanomaterials Institute, and Materials Science and Engineering Department, Drexel University, 3141 Chestnut Street, Philadelphia, PA 19104, United States

${ }^{6}$ College of Engineering and Applied Sciences, Nanjing University, Nanjing 210093, China

${ }^{7}$ School of Chemistry, Physics and Mechanical Engineering Science and Engineering Faculty, Queensland University of Technology, Brisbane, QLD 4001, Australia

KEYWORDS: BN nanosheet, poly (diallyl dimethyl ammonium chloride), composite film, thermal conductivity 


\begin{abstract}
Development of advanced thermal transport materials is a global challenge. Twodimensional nanomaterials have been demonstrated as promising candidates for the thermal management applications. Here, we report a boron nitride $(\mathrm{BN})$ nanosheet/polymer composite film with excellent flexibility and toughness prepared by vacuum-assisted filtration. The mechanical performance of the composite film is highly flexible and robust. It is noteworthy that the film exhibits highly anisotropic properties, with superior in-plane thermal conductivity around $200 \mathrm{~W} \mathrm{~m}^{-1} \mathrm{~K}^{-1}$ and extremely low through-plane thermal conductivity $\left(1.0 \mathrm{~W} \mathrm{~m}^{-1} \mathrm{~K}^{-1}\right)$, making this material an excellent candidate for thermal management in electronics. Importantly, the composite film shows fire-resistance properties. The newly developed unconventional flexible, tough, and refractory $\mathrm{BN}$ films are also promising for heat dissipation in a variety of applications.
\end{abstract}




\section{INTRODUCTION}

The development of nanomaterials with anisotropic thermal transport properties has attracted increasing attention for thermal management applications, such as coatings in electronics and heat sinks. ${ }^{1}$ An ideal candidate should be electrically insulating and have thermal conductivity in-plane significantly greater than through-plane. ${ }^{1}$ Two-dimensional (2D) nanomaterials such as graphene and black phosphorus, both of which possess highly anisotropic thermal conductivity, have recently provided a new platform for addressing heat dissipation in devices. ${ }^{2,3}$ However, it is difficult to employ either in thermal management or build components owing to their electrical conductivity and fast oxidation of phosphorus. BN nanosheets offer a high thermal conductivity, large and direct band gap, resistance to oxidation, high elastic modulus, and a low friction coefficient. $^{4-10} \mathrm{BN}$ nanosheets have been widely explored for use in functional nanocomposite materials, ultraviolet-light emitters, hydrogen storage, and sorbents. ${ }^{11-15}$ In contrast with other conductive and semiconducting 2D materials, such as graphene, transition metal dichalcogenides, and MXenes, BN nanosheets are electrically insulating, which suggests utility in thermal management applications in electronics.

Much effort has recently been devoted to the preparation of highly flexible, strong, and tough graphene, clay, and MXene films for applications in gas separation, molecular- and ion-selective devices, and energy storage. ${ }^{16-20}$ However, pure BN nanosheet films do not offer strength and flexibility required in real-life application. Creating nanocomposite with polymers is an effective approach to improve the mechanical properties of nanomaterials. ${ }^{21,22}$ There have been many studies on BN-polymer nanocomposites with high polymer contents. ${ }^{5,23-25}$ However, the total strength, elongation and thermal conductivities of such composites are largely controlled by the 
polymer, staying far from the inherent properties of $\mathrm{BN}$. To date, some studies have demonstrated that 2D materials such as MXene and graphene-based nanocomposites with low contents of polymer exhibit enhanced mechanical strength and electrical conductivity. ${ }^{16,26,27}$ Therefore, to fully take advantage of the extraordinary properties of these 2D materials, the content of polymer in the composite should be limited. BN nanosheet-based composites with 10 wt\% polyvinyl alcohol (PVA) have recently been fabricated, exhibiting an excellent tensile strength $(125.2 \mathrm{MPa}){ }^{28}$ However, the reported composites show a low thermal conductivity of less than $6.9 \mathrm{~W} \mathrm{~m}^{-1} \mathrm{~K}^{-1}$ owing to the surfactant involved and the thickness of the nanosheet. Therefore, developing a BN/polymer composite film with the flexibility of a polymer, superior thermal conductivity of $\mathrm{BN}$ and the high mechanical strength remains a challenge. Additionally, the fire hazard of polymeric materials restricts their application in thermal management fields, which necessitates the addition of fire resistance. Recent works have shown that graphene oxide and clay can be considered fire resistance while simultaneously improving the mechanical and thermal properties of the composite. ${ }^{29,30}$ This justifies the use BN nanosheets, which are chemically inert and oxidation resistant, ${ }^{31}$ in the construction of multifunctional fire-resistance nanocomposites.

Here, we report a free-standing $\mathrm{BN}$ nanosheet/polymer composite film with a unique combination of properties including flexibility and toughness, anisotropic thermal conductivity, and fire resistance, which is produced by the vacuum-assisted filtration (VAF) of colloidal suspensions of BN nanosheets and polymers (Figure 1a). In this work, poly (diallyl dimethyl ammonium chloride) (PDDA) was chosen, which is a cationic polymer that combines with negatively charged the $\mathrm{BN}$ nanosheet through electrostatic interactions. ${ }^{26}$ Owing to the merits, 
the BN film exhibit highly potential applications in high-performance flexible electrically insulating substrates, superior thermal conductivities and fire-resistance coatings.

\section{EXPERIMENTAL}

Preparation of boron nitride (BN) nanosheets. $B N$ nanosheets were prepared using a previously reported method. ${ }^{32}$ Typically, h-BN powder with the size $2-10 \mu \mathrm{m}$ (Momentive Performance Materials, Inc.) and urea (Sigma-Aldrich) were mixed in a steel milling container using a planetary ball mill (Pulverisette 7, Fritsch). The mixture with a weight ratio of 1:20 (hBN: urea) was protected by nitrogen and milled at a rotation speed of 500 r.p.m. for 20 h. The obtained powders were dissolved in water and dialyzed for 1 week in deionized water to remove the urea, yielding stable aqueous dispersions of BN nanosheets with the size 200-300 nm.

Preparation of BN/PDDA films. BN/PDDA films were fabricated by vacuum-assisted filtration (VAF). To avoid the agglomeration, the BN dispersion $\left(2 \mathrm{mg} \mathrm{mL}^{-1}\right)$ was diluted more than 10 times, then slowly mixed with PDDA solution to form a BN and PDDA dispersion. To form composite films, the mixture was filtered through a glass microfiltration apparatus (Sigma) of ca. $36 \mathrm{~mm}$ as $10,20,30,40,50$, and $60 \mathrm{wt} \%$, and the resulting films were denoted as BN/PDDA 10 , $20,30,40,50$, and $60 \mathrm{wt} \%$, respectively. Pure BN films were produced using a similar approach and were denoted in diameter with a polyethylene membrane under vacuum assistance. These films can be readily peeled off from the polyethylene membrane and retain their freestanding state. The mass fractions of PDDA in the BN and PDDA mixtures were set as BN. ${ }^{32}$

Material characterization. XRD measurements were performed on a PAN alytical X'Pert PRO diffractometer operating with $\mathrm{Cu} \mathrm{K}$ a radiation. SEM analysis was performed on a Zeiss Supra 55 VP SEM instrument. TEM and HRTEM imaging were performed on a JEOL 2100F 
microscope operating at $200 \mathrm{kV}$. The FTIR and optical transmittance spectra were recorded using a Nicolet 7199 FTIR spectrometer and Cary 3 spectrophotometer, respectively. The thermal behavior was analyzed using TGA on a TA Instruments Q50 TGA thermal analyzer at a heating rate of $10{ }^{\circ} \mathrm{C}$ min- 1 from room temperature to $800{ }^{\circ} \mathrm{C}$ under $60 \mathrm{sccm}$ compressed air flow.

Mechanical testing. The BN/PDDA films and BN films were cut into stripes measuring $25 \mathrm{~mm}$ $\times 5 \mathrm{~mm}$ in size and glued onto supporting paper frames. These stripes were fixed in the grips of a universal testing machine (Instron 2360), and then the paper frames were cut and the tensile tests were performed using a $50-\mathrm{N}$ load cell with a cross-head rate of $5 \mathrm{~mm} / \mathrm{min}$. These mechanical tests were conducted at room temperature under a humidity of approximately $45 \%$. The Young's modulus, tensile strength, and strain to failure were calculated as the averages of the results of five parallel experiments.

The hollow cylinders of BN/PDDA 30\% films were produced by rolling the corresponding films $(34 \mathrm{~mm} \times 10 \mathrm{~mm})$ around a glass rod $(6 \mathrm{~mm}$ diameter $)$ and gluing the edges of the strips with a small amount of PDDA solution $(0.1 \mathrm{wt} \%)$. These hollow cylinder specimens were dried in a vacuum oven and weighed, and then they were fixed on a glass slide by PDDA solution (1 $\mathrm{wt} \%$ ) for evaluating the weight loading.

Thermal conductivity. The in-plane thermal conductivity of the BN/PDDA films was measured by a Physical Property Measurement System (PPMS, Quantum Design, USA) using the steadystate method (Figure $\mathrm{S} 1)$. A rectangular $(4 \mathrm{~mm} \times 18 \mathrm{~mm})$ film sample with a radiation shield was placed in a vacuum chamber $\left(9.4 \times 10^{-5}\right.$ Torr $)$ to minimize the radiation, convection, and conduction heat loss. The radiation heat loss is also calculated by the measurement system. A 
heater shoe was connected to one end of the sample, and the other end was fixed to a cold sink. The hot thermometer shoe and cold thermometer shoe were both connected to the measured sample at a distance L (as shown in Figure S1). At steady state, the thermal conductivity (K) of the sample is determined by equation

$K=(Q \times L) /(A \times \Delta T)$, where $Q$ is the net heat flowing through a known cross section $A$ of the measured sample, $\Delta T$ is the temperature difference between the hot thermometer and cold thermometer, and $L$ is the distance between the two thermometer shoes.

The thermal conductivity (through-plane direction) of the BN/PDDA films was analyzed by the device of ai-Phase Mobile 1u using a temperature-wave-analysis method according to ISO 22007-3 (Supplementary Methods). The samples were sandwiched between the heater and sensor plates. By scanning the frequency of heat source, the delay in the phase of the temperature wave was recorded within the range from $-180^{\circ}$ to $-230^{\circ}$ based on an empirical criterion. The linear fitting of phase lag $\theta$ versus square root of frequency, $\operatorname{sqrt}(f)$, was conducted to calculate thermal diffusivity $a$ following equation

$$
\theta=d \times \operatorname{sqrt}(f / a),
$$

where $d$ is the thickness of samples. The thermal conductivity was further calculated based on the thermal diffusivity as well as theoretical density and specific heat capacity of composites. The experimental errors were estimated by means of the square-root-sum error propagation approach, including the following error sources: the measurement of thickness of samples and the physical property measurement system. 
Fire-resistance testing. A parallel flame test was performed on pure PDDA, BN/PDDA 10\% and $30 \%$ films with dimensions of $20 \mathrm{~mm}$ in length and $5 \mathrm{~mm}$ in width. The fire from a gas burner was applied on the tested film for $60 \mathrm{~s}$ and then removed.

\section{RESULTS AND DISCUSSION}

In this work, water-soluble functionalized boron nitride nanosheet having negative surface charge and sizes of around 200-300 nm were prepared according to our previously reported method (Figure S2). ${ }^{32}$ The negatively-charged BN nanosheets attracts positively-charged PDDA through electrostatic interactions, producing a uniform hybrid structure. The BN/PDDA composite films can be easily peeled off from the filter membrane after filtration to form freestanding paper-like laminated films. The thickness and size of the BN/PDDA composite films can be easily adjusted by the feed amounts of the colloidal suspensions of the BN nanosheets and PDDA and the size of the filtration system, respectively.

The films are nearly transparent (Figure 1a and Figures S3), smooth, and flexible and can be readily processed into desired shapes (Figure 1b). The transparency of the films was further demonstrated using UV-Vis spectroscopy (Figure S4). These composite films can be easily reshaped and made more flexible by increasing the content of PDDA. This is in stark comparison to the pristine $\mathrm{BN}$ film, which is weak and cannot be folded into desired shapes. The flexibility of the BN/PDDA film mainly comes from the strong interactions of the BN nanosheets with the PDDA through electrostatic interactions (Figure 1c).

SEM and TEM were used to characterize the morphology of the films. The pure PDDA film is homogenous, while the BN/PDDA films are composed of parallel-stacked layers (Figure 1d-f). The BN nanosheets and PDDA chains in the composite films are alternating, indicating that they 
disperse in the aqueous solution homogenously and form compact films when filtered, as shown in the TEM image (Figure $1 \mathrm{~g}$ and Figure S5). In addition, the spacing between the layers decreases with decreasing amounts of PDDA to $10 \mathrm{wt} \%$ (Figure S6) indicating the intercalation effects of PDDA into BN layers.
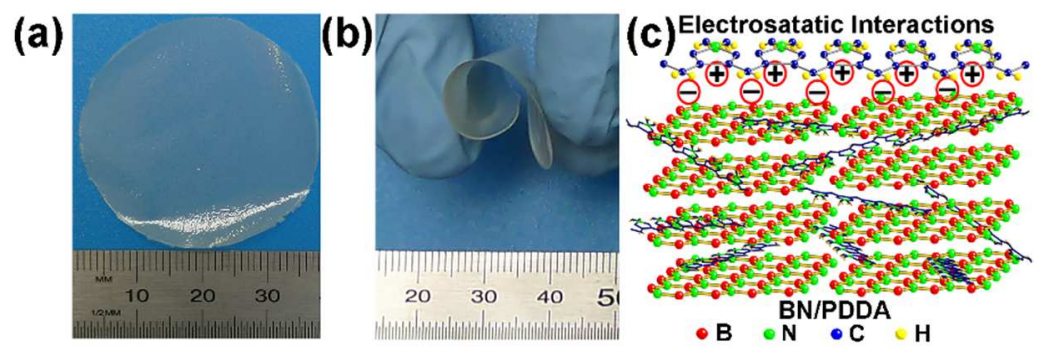

(d)

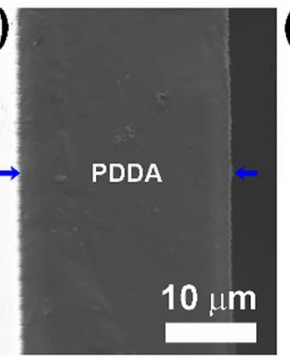

(e)
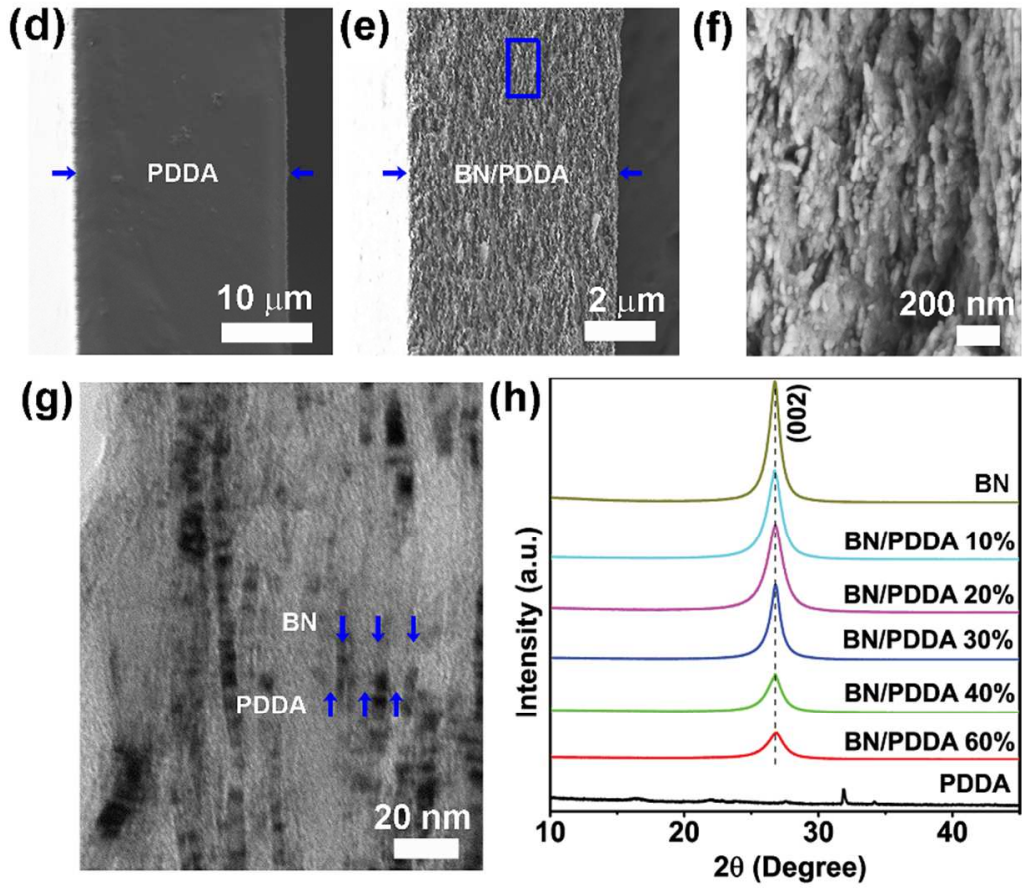

Figure 1. (a) Photo of a free-standing BN/PDDA 30\% film. (b) Photo of a scrolled BN/PDDA $30 \%$ film. (c) Schematic illustration of composite film preparation by VAF. (d) Cross-sectional SEM images of PDDA and (e, f) BN/PDDA 30\% films. (g) Cross-sectional TEM image of BN/PDDA 30\% film. (h) XRD patterns of BN/PDDA films. 
The structures of the BN/PDDA films were further analyzed by X-ray diffraction (XRD). One characteristic diffraction peak can be observed at $26.2^{\circ}$, which arises from the (002) plane of BN (Figure 1h). With the increasing incorporation of PDDA, the (002) peak shifts to a higher diffraction angle and exhibits a remarkably reduced intensity, indicating that the spacing between the layers of BN slightly decreases with the increasing amount of PDDA owing to the stacking force from the PDDA, but that fewer stacked BN layers are present. PDDA layers separate most flakes. This is consistent with the TEM results and further demonstrates that the PDDA can enter into the space between $\mathrm{BN}$ nanosheets. ${ }^{26,33}$

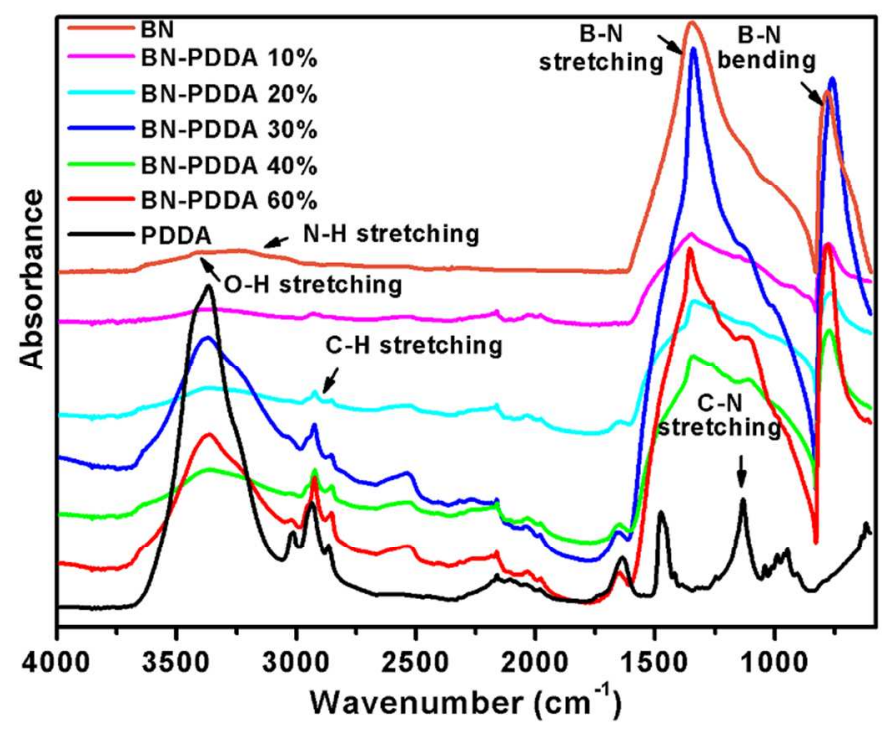

Figure 2. FTIR spectra of PDDA, BN/PDDA10\%, 20\%, 30\%, 40\%, 50\%, 60\% and pure BN films.

The Fourier-transform infrared (FTIR) spectrum of the BN/PDDA films (Figure 2) show the two strong characteristic absorption bands of BN nanosheets of the in-plane B-N stretching vibrations at approximately $1360 \mathrm{~cm}^{-1}$ and the out-of-plane B-N-B bending vibrations at approximately $755 \mathrm{~cm}^{-1} \cdot{ }^{34}$ Moreover, a broad peak from $3000 \sim 3600 \mathrm{~cm}^{-1}$ can been seen in the 
inset due to the N-H- (approximately $3250 \mathrm{~cm}^{-1}$ ) and $\mathrm{O}-\mathrm{H}-\left(\right.$ approximately $3410 \mathrm{~cm}^{-1}$ ) stretching vibrations from the fabricating process ${ }^{38,39}$. The pure PDDA film shows only the characteristic C-H stretching vibrations at $2973 \mathrm{~cm}^{-1}, \mathrm{C}-\mathrm{N}$ stretching vibrations at approximately $1124 \mathrm{~cm}^{-1}, 35$ and bands from 3150 to $3550 \mathrm{~cm}^{-1}$ that are due to the $\mathrm{O}-\mathrm{H}$ stretching vibrations of water absorbed in the PDDA chains. ${ }^{36}$

The $-\mathrm{NH}_{2}$ and $-\mathrm{OH}$ groups can also be confirmed by an X-ray photoelectron spectroscopy (XPS). As shown in Figure S7a, the peak at $190.4 \mathrm{eV}$ corresponds to B-N bonds in BN, and the shoulder $(191.2 \mathrm{eV})$ should be attributed to the $\mathrm{B}$ atoms in $\mathrm{B}-\mathrm{O}$ bonds formed due to the $-\mathrm{OH}$ groups attached to defects along the edges in $\mathrm{BN} .{ }^{37}$ In Figure $\mathrm{S} 7 \mathrm{~b}$, the main peak with a binding energy of $398 \mathrm{eV}$ in the $\mathrm{N}$ 1s spectrum corresponds to $\mathrm{N}-\mathrm{B}$ bonds in $\mathrm{BN}$, a shoulder can be deconvoluted at $399 \mathrm{eV}$ corresponding to $\mathrm{N}-\mathrm{H}$ bonds which further confirmed the presence of $\mathrm{NH}_{2}$ group. $^{32}$

Furthermore, the BN before and after exfoliation exhibit high negative potential value, -44 and $-33 \mathrm{mV}$, respectively. ${ }^{32}$ The BN nanosheets exhibits a somewhat decreased absolute value of zeta potential $(-33 \mathrm{mV})$ after functionalization. The reduced negative zeta potential after functionalization is due to the partial neutralization effect between positively charged $\mathrm{NH}^{3+}$ groups and negatively charged oxygen containing groups formed on BN surface upon contact with water. ${ }^{32}$

Unlike other 2D films, including MXene, graphene oxide and carbon nanotube-based films, ${ }^{26,37,38}$ which exhibit good mechanical properties, pure BN nanosheet films are usually brittle and difficult to handle due to a small lateral size of BN flakes. The Young's modulus and tensile strength of the $\mathrm{BN}$ films are $28.3 \pm 10.6$ and $7 \pm 0.4 \mathrm{MPa}$, respectively (Figure 3a and 
Supplementary Table S1). These values are rather low compared with those of the 2D films mentioned above and far below that of single-crystal hBN ( $\sim 36.5 \mathrm{GPa})$ due to weak bonding between the multi-layer BN sheets in the films. ${ }^{39}$ However, upon introducing PDDA, the Young's modulus and tensile strength are greatly improved. As shown in Figure 3a, Figure S8 and Table S1, upon introducing $10 \mathrm{wt} \%$ PDDA into the BN nanosheets films, the Young's modulus and tensile strength are improved by approximately 10 times, respectively. The strain to failure is also enhanced by the addition of more PDDA (Figure S9). The mechanical properties of other BN/PDDA films with different polymer contents are shown in Figure 3a,b, indicating that the mechanical properties of the composite can be tailored by changing the ratio of $\mathrm{BN}$ nanosheets to PDDA. The dramatic enhancements in the strength, modulus, and strain to failure of these composite films originated from three main reasons: (i) a good dispersion of BN nanosheets ${ }^{32}$ in the polymer matrix due to the $-\mathrm{NH}_{2}$ and $-\mathrm{OH}$ groups on their surface; (ii) these functional groups could increase the interfacial interaction between BN nanosheets and the PDDA through the electrostatic interactions in BN/PDDA. As we reported before, the zeta potential of $\mathrm{BN}$ is $-33 \mathrm{mV} .^{32}$ Therefore, the $\mathrm{BN}$ nanosheets with negative surface charge attract positively charged PDDA, which is similar with the previous report ${ }^{40}$ (Figure 1c). When external stress is applied to a BN/PDDA composite film, it can easily be transferred from a nanosheet to a polymer and from the PDDA to another nanosheet due to the strong interfacial interactions; ${ }^{41}$ (iii) intrinsic properties of $\mathrm{BN}$ are largely maintained in the films produced by vacuum-assisted filtration and lead to the inherent damage resistance of the composite films. In summary, BN/PDDA films can offer superior mechanical properties and find many applications. 
(a)

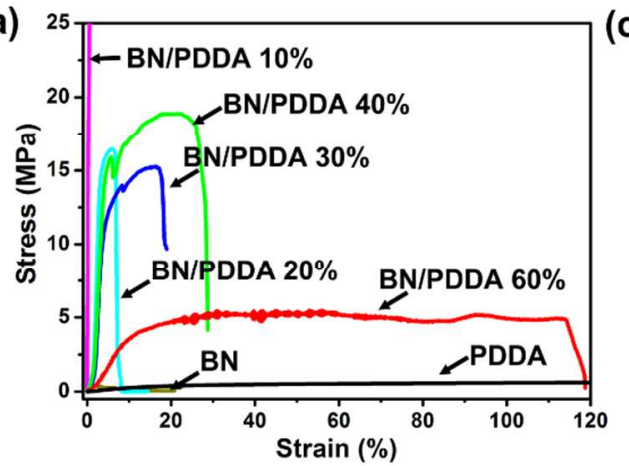

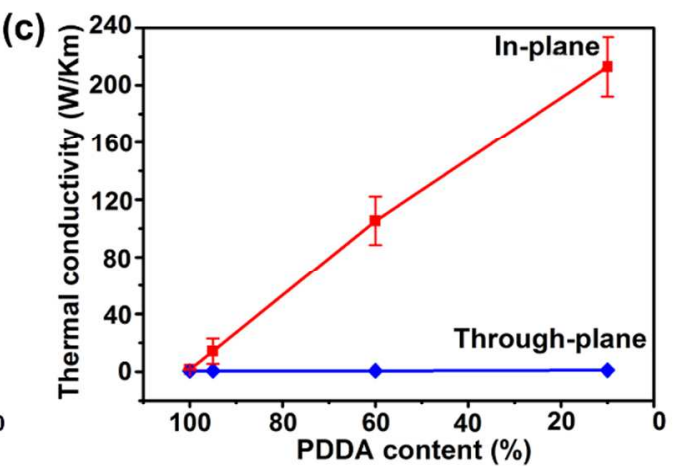

(b)

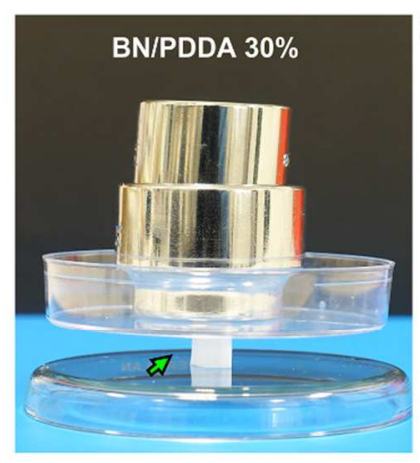

(d) 250

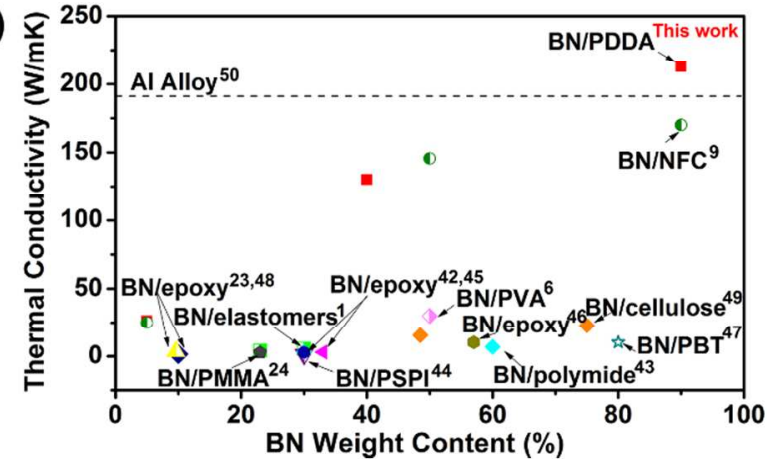

Figure 3. (a) Stress-strain curves of BN, BN/PDDA, and PDDA films. (b) A BN/PDDA 30\% film supporting $\sim 17,000$ times its own weight. (c) In-plane and through-plane thermal conductivities of BN/PDDA films with different contents of PDDA. (d) Thermal conductivity enhancement of the BN/PDDA composites reported in previous works.

To observe the compressive strengths of the BN/PDDA films, we used hollow cylinders that were made by rolling the BN/PDDA films and pasting the overlapping edges with PDDA. ${ }^{26}$ As shown in Figure 3b, a hollow cylinder of BN/PDDA 30\% (6 mm in diameter and $10 \mathrm{~mm}$ high), made from a film of $34 \mathrm{~mm}$ in length and $10 \mathrm{~mm}$ in width can readily support approximately 17,000 times its own weight without suffering visible deformation, suggesting that their compressive strength is very high. The robust compressive strength and toughness of the composite films further demonstrate the strong interfacial interactions between BN nanosheets and polymers through the electrostatic interactions. The high compressive strength and toughness, 
together with the exceptional flexibility, suggest that these BN nanosheet-based composite films may find applications as structural or functional materials in thermally conductive actuators, flexible energy storage devices, and temperature sensors. ${ }^{16,42}$

The BN/PDDA films not only exhibit the enhanced mechanical properties of the BN but also show highly anisotropic thermal conductivity. The thermal conductivities of BN/PDDA films with different PDDA weight percentages are presented in Figure 3c. It can be seen that the inplane thermal conductivity increases with the decreasing weight fraction of PDDA. The in-plane thermal conductivity can be up to $212.8 \mathrm{~W} \mathrm{~m}^{-1} \mathrm{~K}^{-1}$ for BN/PDDA $10 \%$ films. Even for $\mathrm{BN} / \mathrm{PDDA} 60 \%$ films, the in-plane thermal conductivity values can reach $105.2 \mathrm{~W} \mathrm{~m}^{-1} \mathrm{~K}^{-1}$, respectively. In order to demonstrate the superiority thermal conductivity of the BN/PDDA film, Figure $3 \mathrm{~d}$ summarizes previously reported thermal conductivity of BN/polymer composites with different BN weight contents in the polymer matrix. ${ }^{1,6,9,23,24,43-50}$ One can see that the BN/PDDA film exhibits the highest thermal conductivity enhancement with the similar BN weight content among the reported BN/polymer composites. Moreover, BN/PDDA $10 \%$ is superior to conventional airspace materials such as aluminum alloy ${ }^{51}$ and comparable to aluminum metal. It is notable that the BN/PDDA films exhibit a large anisotropy of thermal conductivity with an extremely low through-plane thermal conductivity $\left(1.0 \mathrm{~W} \mathrm{~m}^{-1} \mathrm{~K}^{-1}\right)$. The anisotropy of the thermal conductivity is due to the layered structure consisting of the $\mathrm{BN}$ nanosheets aligned in the polymers due to self-assembly or BN nanosheets bonded by very thin layers of polymer at the high $\mathrm{BN}$ contents. The heat transfer strongly depends on the orientation of 2D nanomaterials. ${ }^{52}$ The $\mathrm{BN}$ nanosheets were evenly distributed in the cross-section and aligned parallel to the surface of the films due to the good dispersion of BN nanosheets in the PDDA matrix. Therefore, there is a large contact area between the adjacent BN nanosheets (Figure 1e-g, 

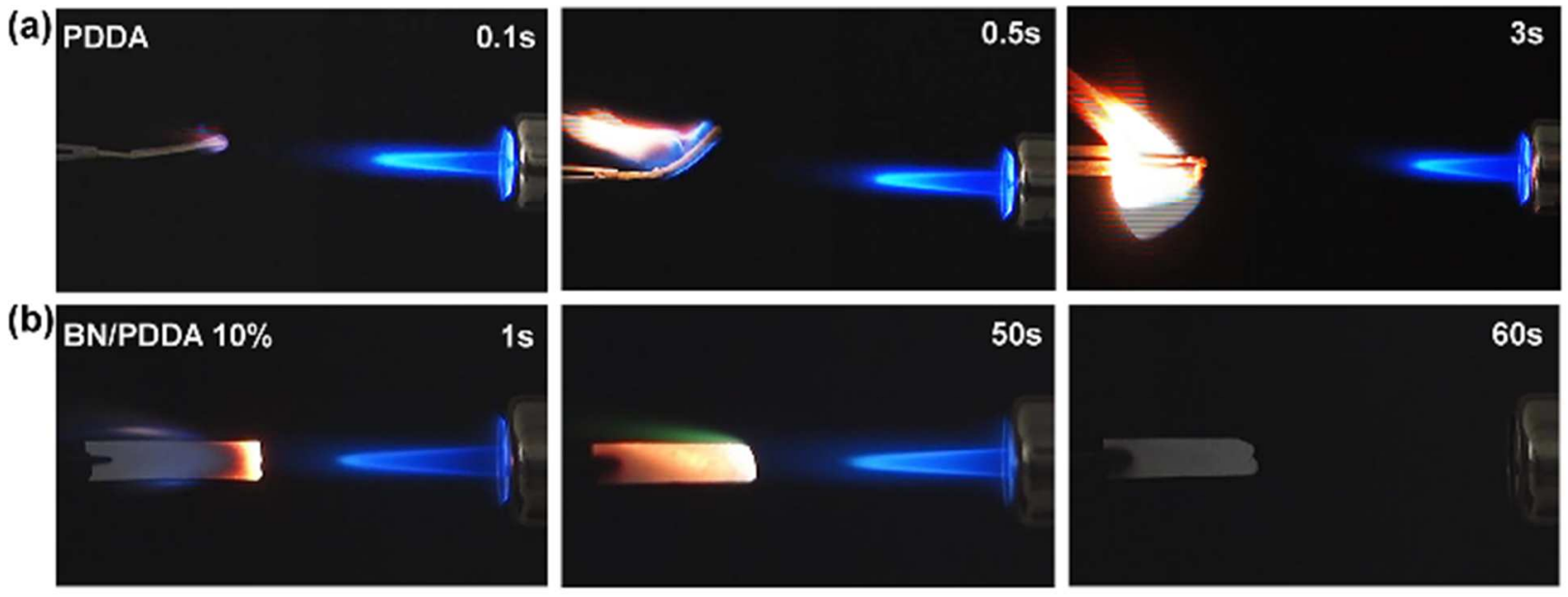

Figure 4. (a) Photographs of pure PDDA and (b) BN/PDDA $10 \%$ films on a hot flame for different times.

Many of the commonly used flame resistance are organic materials, which often show poor tolerance to oxidants with negative environmental and health effects. ${ }^{54}$ However, recent work has shown that the fire-resistance of materials can be improved by the addition of $2 \mathrm{D}$ materials such as clays and graphene oxides. In addition, it is known that $\mathrm{BN}$ materials show high resistance to oxidation. ${ }^{15}$ Nanostructured $\mathrm{BN}$ is therefore an ideal candidate in the construction of multifunctional fire-resistance polymer nanocomposites. Here, BN/PDDA films with outstanding 
electrical insulation, high thermal conductivity, good chemical stability, and oxidation resistance are promising candidates for the development of new types of fire-resistance materials. Parallel burning tests (Figure 4, and Figure S11) show that BN/PDDA 10-30\% films display excellent fire-resistance, where the films can nearly maintain their initial shape after burning for more than $60 \mathrm{~s}$. The results indicate that the flame-resistance BN nanosheets disperses in polymer matrix homogenously which enables the preservation of the flame-resistance functions of films. However, the pure PDDA films ignited on the flame and were burned out within 3s (Figure 4). Thermogravimetric analysis (TGA) in air revealed that the oxidation resistance of the BN/PDDA films increases with the BN content (Figure S11b). The superior flame-resistance property of the BN/PDDA films may significantly improve safety when they are used as heat shields, flexible fire-resistant coatings, and electrically insulating substrates. To demonstrate the potential application of the BN/PDDA films in cooling electronic device, the BN/PDDA $30 \%$ film is used as a substrate with a size of $40 \times 40 \mathrm{~mm}$ to fix one light-emitting-diode (LED) chip on the central of sample (Figure 5a,b). Silver paste was used for adhesion between the LED chip and samples. Polyimides (PI) abstract was selected as a comparison sample, because it has been used widely in printed circuit boards due to the lightweight and high mechanical toughness. When the PI was as the substrate, after steady-state $(10 \mathrm{~min})$ of the heat flow caused by the LED chip, a hot spot appeared at the center $\left(75^{\circ} \mathrm{C}\right)$, with a high temperature gradient from the center to the edge (Figure 5c) due to the poor thermal conductivity of the PIs $\left(0.3 \mathrm{~W} \mathrm{~m}^{-1} \mathrm{~K}^{-1}\right)$. However, the BN/PDDA $30 \%$ film exhibits a dramatically reduced center spot temperature $\left(52^{\circ} \mathrm{C}\right)$ with a more uniform temperature distribution under similar conditions (Figure 5d). The BN/PDDA film exhibits highly anisotropic properties as confirmed by the thermal conductivity measurement (Figure 5c). It indicates that the heat is not being conducted well in the through-plane direction, 
(a)

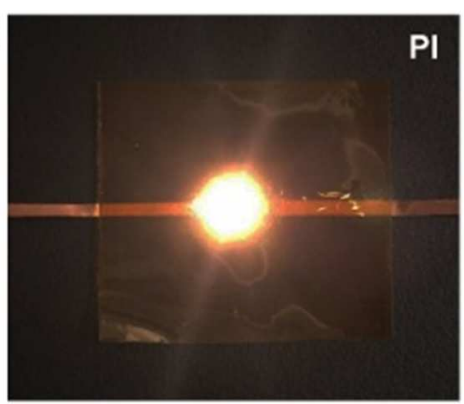

(c)

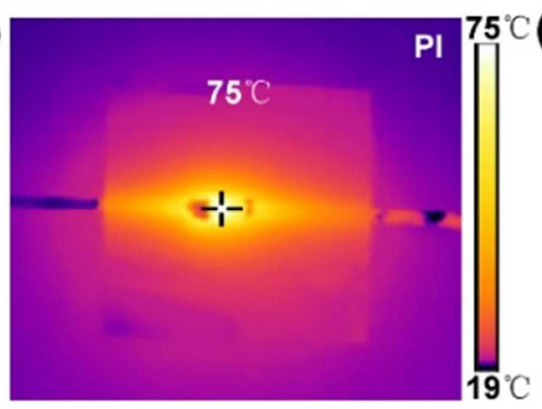

(e)

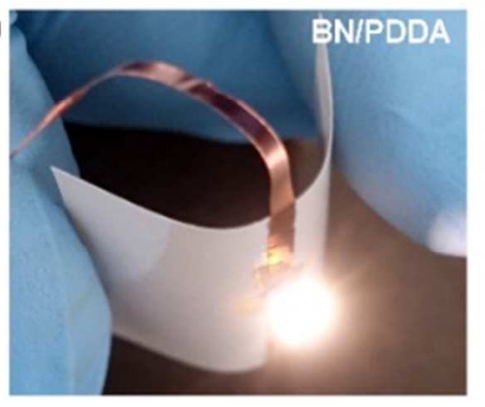

(b)
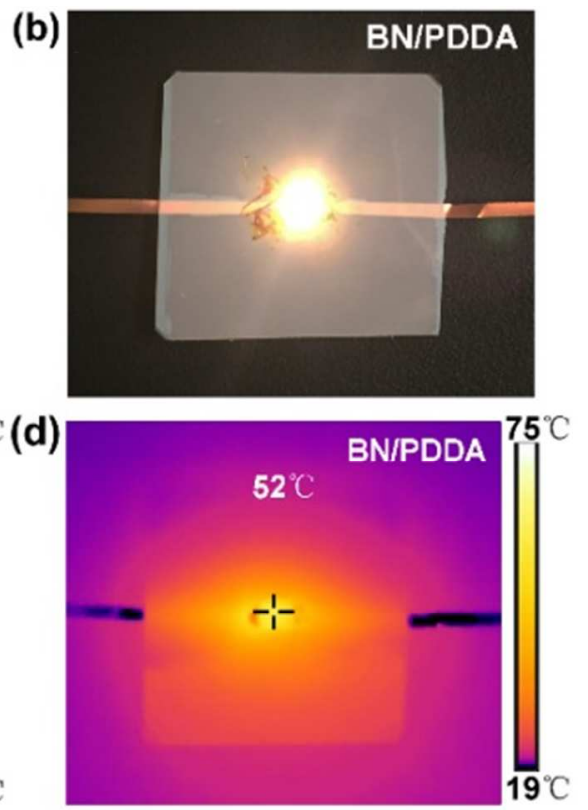

(f)

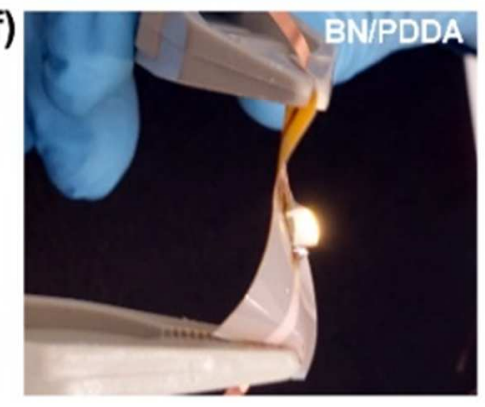

Figure 5. Application of the BN/PDDA 30\% film in flexible substrates. Photos of the PI (a) and BN/PDDA $30 \%$ film substrates (b). The corresponding thermal images of the PI (c) and BN/PDDA 30\% film (d). (e, f) Photos of working electronic devices by using a BN/PDDA 30\% film substrate, exhibiting its excellent flexibility.

\section{CONCLUSIONS}


In summary, nearly transparent, flexible and tough BN/PDDA films have been manufactured by simple and scalable vacuum assisted filtration. The Young's modulus, tensile strength, and strain to failure of the $\mathrm{BN}$ films improved dramatically after incorporation of PDDA. The composite films exhibit highly anisotropic thermal conductivity reaching $\sim 200 \mathrm{~W} \mathrm{~m}^{-1} \mathrm{~K}^{-1}$ in plane and being $\sim 1.0 \mathrm{~W} \mathrm{~m}^{-1} \mathrm{~K}^{-1}$ in the out-of-plane direction. Those properties combined with high chemical and temperature stability make them useful for various applications, particularly in thermal management (heat spreading), and flexible and fire-resistant coatings.

\section{ASSOCIATED CONTENT}

\section{Supporting Information.}

Illustration of the thermal conductivity setup, additional photos, optical transmittance, FTIR, mechanical properties, TGA of BN/PDDA films. This material is available free of charge via the Internet at http://pubs.acs.org.

\section{AUTHOR INFORMATION}

\section{Corresponding Author}

* Corresponding author E-mail: weiwei.lei@deakin.edu.au

\section{Author Contributions}

The manuscript was written through contributions of all authors. All authors have given approval to the final version of the manuscript. 


\section{Funding Sources}

This work was financially supported by the National Natural Science Foundation of China (No. 51304166), the Australian Research Council Discovery Program, the Australian Re-search Council Discovery Early Career Researcher Award scheme (DE150101617 and DE140100716), and Deakin University Central Research Grants Scheme.

\section{Notes}

The authors declare no competing financial interests

\section{ACKNOWLEDGMENT}

We thank R. V. Driel for helping us to prepare the samples for HRTEM measurements.

\section{REFERENCES}

(1) Kuang, Z.; Chen, Y.; Lu, Y.; Liu, Y.; Hu, S.; Wen, S.; Mao. Y; Zhang. L.; Fabrication of highly oriented hexagonal boron nitride nanosheet/elastomer nanocomposites with high thermal conductivity. Small 2015, 11, 1655-1659.

(2) Renteria, J. D.; Ramirez, S.; Malekpour, H.; Alonso, B.; Centeno, A.; Zurutuza, A.; Cocemasov, A. I.; Nika, D. L.; Balandin, A. A. Strongly anisotropic thermal conductivity of free-standing reduced graphene oxide films annealed at high temperature. Adv. Funct. Mater. 2015, 25, 4664-4672.

(3) Lee, S.; Yang, F.; Suh, J.; Yang, S.; Lee, Y.; Li, G.; Choe, H. S.; Suslu, A.; Chen, Y.; Ko, C.; Park, J.; Liu, K.; Li, J.; Hippalgaonkar, K.; Urban, J. J.; Tongay, S.; Wu, J. Anisotropic inplane thermal conductivity of black phosphorus nanoribbons at temperatures higher than $100 \mathrm{~K}$. Nat. Commun. 2015, 6, 8573. 
(4) Sichel, E. K.; Miller, R. E.; Abrahams, M. S.; Buiocchi, C. J. Heat capacity and thermal conductivity of hexagonal pyrolytic boron nitride. Phys. Rev. B 1976, 13, 4607-4611.

(5) Zhi, C.; Bando, Y.; Tang, C.; Kuwahara, H.; Golberg, D. Large-scale fabrication of boron nitride nanosheets and their utilization in polymeric composites with improved thermal and mechanical properties. Adv. Mater. 2009, 21, 2889-2893.

(6) Song, W.; Wang, P.; Cao, L.; Anderson, A.; Meziani, M. J.; Farr, A. J Polymer/boron nitride nanocomposite materials for superior thermal transport performance. Angew. Chem. Int. Ed. 2012, 51, 6498-6501.

(7) Liu, D.; Lei, W.; Qin, S.; Klika, K. D.; Chen, Y. Superior adsorption of pharmaceutical molecules by highly porous BN nanosheets. Phys. Chem. Chem. Phys. 2016, 18, 84-88.

(8) Lei, W.; Liu, D.; Chen, Y. Highly crumpled boron nitride nanosheets as adsorbents: scalable solvent-less production. Adv. Mater. Interfaces 2015, 2, 2-7.

(9) Zhu, H.; Li, Y.; Fang, Z.; Xu, J.; Cao, F.; Wan, J.; Preston, C.; Yang, B.; Hu, L. Highly thermally conductive papers with percolative layered boron nitride nanosheets. ACS Nano 2014, 8, 3606-3613.

(10) Golberg, D.; Bando, Y.; Huang, Y.; Terao, T.; Boron nitride nanotubes and nanosheets. ACS Nano 2010, 4, 2979-2993.

(11) Lei, W.; Zhang, H.; Wu, Y; Zhang, B.; Liu, D.; Qin, S.; Liu, Z.; Liu, L.; Ma, Y.; Chen, Y. Oxygen-doped boron nitride nanosheets with excellent performance in hydrogen storage. Nano Energy 2014, 6, 219-224.

(12) Liu, D.; Lei, W.; Qin, S.; Chen, Y. Template-free synthesis of functional 3D BN architecture for removal of dyes from water. Sci. Rep. 2014, 4, 4453. 
(13) Liu, D.; He, L.; Lei, W.; Klika, K. D.; Kong, L.; Chen, Y. Adv. Mater. Interfaces Multifunctional polymer/porous boron nitride nanosheet membranes for superior trapping emulsified oils and organic molecules. Adv. Mater. Interfaces 2015, 2, 1-6.

(14) Watanabe, K.; Taniguchi, T.; Kanda, H. Direct-bandgap properties and evidence for ultraviolet lasing of hexagonal boron nitride single crystal. Nat. Mater. 2004, 3, 404-409.

(15) Lei, W.; Portehault, D.; Liu, D.; Qin, S.; Chen, Y. Porous boron nitride nanosheets for effective water cleaning. Nat. Commun. 2013, 4, 1777.

(16) Zhang, M.; Huang, L.; Chen, J.; Li, C.; Shi, G. Ultratough , ultrastrong , and highly conductive graphene films with arbitrary sizes. Adv. Mater. 2014, 26, 7588-7592.

(17) Li, C. Y.; Schulz, J.; Mannen, S.; Delhom, C.; Condon, B.; Chang, S.; Zammarano, M.; Grunlan, J. C. Flame retardant behavior of polyelectrolyte-clay thin film assemblies on cotton fabric. ACS Nano 2010, 4, 3325-3337.

(18) Gogotsi, Y. Chemical Vapour Deposition: Transition Metal Carbides Go 2D. Nat. Mater. 2015, 14, 1079-1080.

(19) Wan, W.; Zhao, Z.; Hu, H.; Gogotsi, Y.; Qiu, J. Highly controllable and green reduction of graphene oxide to flexible graphene film with high strength. Mater. Res. Bull. 2013, 48, 47974803.

(20) Joshi, R. K.; Carbone, P.; Wang, F. C.; Kravets, V. G.; Su, Y.; Grigorieva, I. V.; Wu, H. A.; Geim, A. K.; Nair, R. R. Precise and ultrafast molecular sieving through graphene oxide membranes. Science. 2014, 343, 752-754.

(21) Yan, Y. X.; Yao, B. H.; Mao, L. B.; Asiri, A. M.; Alamry, K. A.; Marwani, H. M.; Yu, S. H. Micrometer-thick graphene oxide-layered double hydroxide nacre-inspired coatings and their properties. Small 2016, 12, 745-755. 
(22) Zhang, J.; Feng, W.; Zhang, H.; Wang, Z.; Calcaterra, H. A.; Yeom, B.; Hu, P. A.; Kotov, N. A. Multiscale deformations lead to high toughness and circularly polarized emission in helical nacre-like fibres. Nat. Commun. 2016, 7, 10701.

(23) He, Y. M.; Wang, Q. Q.; Liu, W.; Liu, Y. S. Functionalization of boron nitride nanoparticles and their utilization in epoxy composites with enhanced thermal conductivity. Phys. Status Solidi A 2014, 211, 677-684.

(24) Wang, X.; Pakdel, A.; Zhang, J.; Weng, Q.; Zhai, T.; Zhi, C.; Golberg, D.; Bando, Y. Largesurface-area $\mathrm{BN}$ nanosheets and their utilization in polymeric composites with improved thermal and dielectric properties. Nanoscale Res. Lett. 2012, 7, 662.

(25) Wang, S.; Li, Q.; Zhang, S.; Pan, L. Tribological behavior of poly (phenyl phydroxybenzoate)/polytetrafluoroethylene composites filled with hexagonal boron nitride under dry sliding condition. Mater. Des. 2013, 43, 507-512.

(26) Ling, Z.; Ren, C. E.; Zhao, M. Q.; Yang, J.; Giammarco, J. M.; Qiu, J.; Barsoum, M. W.; Gogotsi, Y. Flexible and conductive MXene films and nanocomposites with high capacitance. Proc. Natl. Acad. Sci. 2014, 111, 16676-16681.

(27) Li, Y. Q.; Yu, T.; Yang, T. Y.; Zheng, L. X.; Liao, K. Bio-inspired nacre-like composite films based on graphene with superior mechanical, electrical, and biocompatible properties. $A d v$. Mater. 2012, 24, 3426-3431.

(28) Zeng, X.; Ye, L.; Yu, S.; Li, H.; Sun, R.; Xu, J.; Wong, C. Artificial nacre-like papers based on noncovalent functionalized boron nitride nanosheets with excellent mechanical and thermally conductive properties. Nanoscale 2015, 7, 6774-6781. 
(29) Wicklein, B.; Kocjan, A.; Salazar, A. G.; Carosio, F.; Camino, G.; Antonietti, M.; Bergström, L. Thermally insulating and fire-retardant lightweight anisotropic foams based on nanocellulose and graphene oxide. Nat. Nanotechnol. 2015, 10, 277-283.

(30) Fang, B.; Peng, L.; Xu, Z.; Gao, C. Wet-spinning of continuous montmorillonite-graphene fibers for fire-resistant lightweight conductors. ACS Nano 2015, 9, 5214-5222.

(31) Gogotsi, Y.; Lavrenko, V. A. Corrosion of High-Performance Ceramics (Springer, Berlin) 1992, 190.

(32) Lei, W.; Mochalin, V. N.; Liu, D.; Qin, S.; Gogotsi, Y.; Chen, Y. Boron nitride colloidal solutions, ultralight aerogels and freestanding membranes through one-step exfoliation and functionalization. Nat. Commun. 2015, 6, 8849.

(33) Xie, B. H.; Huang, X.; Zhang, G. J. High thermal conductive polyvinyl alcohol composites with hexagonal boron nitride microplatelets as fillers. Compos. Sci. Technol. 2013, 85, 98-103.

(34) Zhi, C.; Bando, Y.; Tang, C.; Golberg, D.; Xie, R.; Sekiguchi, T. Large-scale fabrication of boron nitride nanohorn. Appl. Phys. Lett. 2005, 87, 063107.

(35) Wang, S.; Yu, D.; Dai, L. Polyelectrolyte functionalized carbon nanotubes as efficient metal-free electrocatalysts for oxygen reduction. J. Am. Chem. Soc. 2011, 133, 5182-5185.

(36) Yang, D.; Rochette, J.; Sacher, E. Spectroscopic evidence for $\pi-\pi$ interaction between poly (diallyl dimethylammonium) chloride and multiwalled carbon nanotubes. J. Phys. Chem. B. 2005, 62, 4481-4484.

(37) Dikin, D.; Stankovich, S.; Zimney, E. J.; Piner, R. D.; Dommett, G. H.; Evmenenko, G.; Nguyen, S. T.; Ruoff, R. S. Preparation and characterization of graphene oxide paper. Nature 2007, 448, 457-460. 
(38) Li, Z.; Xu, J.; Byrne, J. P.; Chen, L.; Wang, K.; Morris, M. A.; Holmes, J. D. Freestanding bucky paper with high strength from multi-wall carbon nanotubes. Mater. Chem. Phys. 2012, 135, 921-927.

(39) Solozhenko, V. L.; Will, G.; Elf, F. Isothermal compression of hexagonal graphite-like boron nitride up to $12 \mathrm{GPa}$. Solid State Commun. 1995, 96, 1-3.

(40) Liu, D.; Zhang, M. W. He, L.; Chen, Y.; Lei, W. W. Layer-by-layer assembly fabrication of porous boron nitride coated multifunctional materials for water cleaning. Adv. Mater. Interfaces 2017, 4, 1700392.

(41) Khan, U.; May, P.; Neill, A. O.; Bell, A. P.; Boussac, E.; Martin, A.; Coleman, J. N. Polymer reinforcement using liquid-exfoliated boron nitride nanosheets. Nanoscale 2013, 5, $581-587$.

(42) Li, N.; Chen, Z.; Ren, W.; Li, F.; Cheng, H. M. Flexible graphene-based lithium ion batteries with ultrafast charge and discharge rates. Proc. Natl. Acad. Sci. 2012, 109, 1736017365.

(43) Huang, X.; Zhi, C.; Jiang, P.; Golberg, D.;Bando, Y.; Tanaka, T. Polyhedral oligosilsesquioxane-modified boron nitride nanotube based epoxy nanocomposites: an ideal dielectric material with high thermal conductivity. Adv. Funct. Mater. 2013, 23, 1824-1831.

(44)Sato, K.; Horibe, H.; Shirai, T.; Hotta, Y.; Nakano, H.; Nagai, H.; Mitsuishi, K.; Watari, K. Thermally conductive composite films of hexagonal boron nitride and polyimide with affinityenhanced interfaces. J. Mater. Chem. 2010, 20, 2749-2752.

(45) Li, T. L.; Hsu, S. L. Preparation and properties of thermally conductive photosensitive polyimide/boron nitride nanocomposites. J. Appl. Polym. Sci. 2011, 121, 916-922. 
(46) Wattanakul, K.; Manuspiya, H.; Yanumet, N. Effective surface treatments for enhancing the thermal conductivity of BN - filled epoxy composite. J. Appl. Polym. Sci. 2011, 119, 3234-3243.

(47) Xu, Y. S.; Chung, D. D. Vertically and compactly rolled-up reduced graphene oxide film/epoxy composites: a two-stage reduction method for graphene-based thermal interfacial materials. Compos. Interfaces 2000, 7, 243-256.

(48) Morishita, T.; Okamoto, H. Facile exfoliation and noncovalent superacid functionalization of boron nitride nanosheets and their use for highly thermally conductive and electrically insulating polymer nanocomposites. ACS Appl. Mater. Interfaces 2016, 8, 27064-27073.

(49) Chen, J.; Huang, X.; Zhu, Y.; Jiang, P. Cellulose nanofiber supported 3D interconnected BN nanosheets for epoxy nanocomposites with ultrahigh thermal management capability. Adv. Funct. Mater. 2017, 27, 1604754.

(50) Nagaoka, S.; Jodaibd, T.; Kameyamac, Y.; Horikawaacd, M.; Shirosakiad, T.; Ryuad, N.; Takafujicd, M.; Sakuraicd, H.; Iharacd, H. Cellulose/boron nitride core-shell microbeads providing high thermal conductivity for thermally conductive composite sheets. $R S C A d v . \mathbf{2 0 1 6}$, 6, 33036-33042.

(51) Male, A. T.; Chen, Y. W.; Pan, C.; Zhang, Y. M. Rapid prototyping of sheet metal components by plasma-jet forming. J. Mater. Process. Technol. 2003, 135, 340-346.

(52) Han, D.; Zhao, Y. H.; Zhang, Y. F.; Bai, S. L. Vertically and compactly rolled-up reduced graphene oxide film/epoxy composites: a two-stage reduction method for graphene-based thermal interfacial materials. RSC Advances 2015, 5, 94426-94435.

(53) Han, W. Anisotropic hexagonal boron nitride nanomaterials: synthesis and applications, Nanotechnologies Life Sci. 2008, 41, 1-60. 
(54) Hale, R. C.; Guardia, M. J. L.; Harvey, E. P.; Gaylor, M. O.; Matteson, T.; Mainor, M.; Duff, W; H. Flame retardants: Persistent pollutants in land-applied sludges. Nature 2001, 412, $140-141$.

TOC
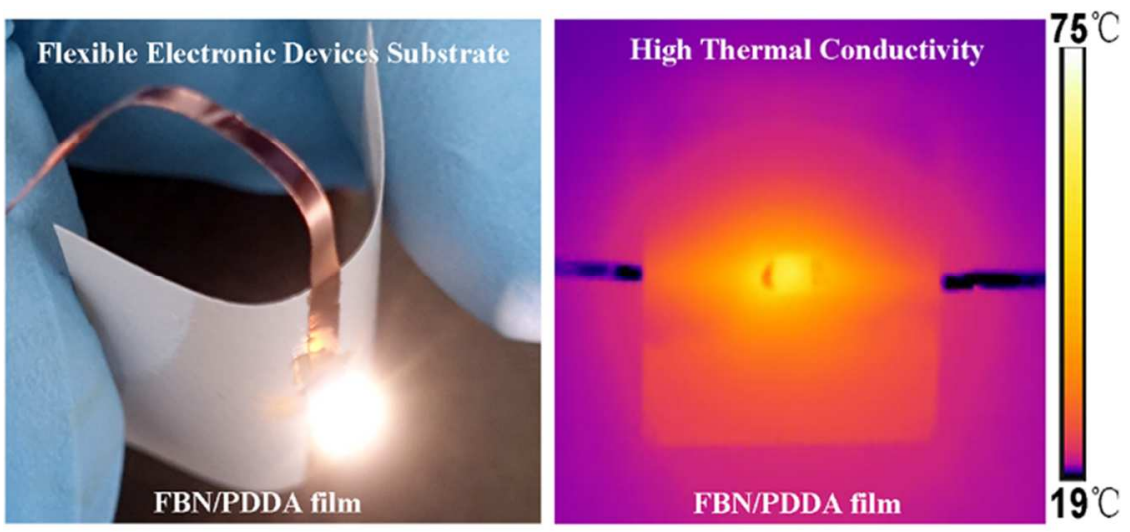

ACS Paragon Plus Environment 